\title{
m-tutorial: ferramenta de autoria para desenvolvimento de tutoriais voltados para o m-learning
}

Title: m-tutorial: An Authoring Tool for m-learning Tutorial Development

Christien Lana Rachid

Pontifícia Universidade Católica de Minas Gerais

Unidade São Gabriel, Belo Horizonte, MG - Brasil

christienrachid@gmail.com

\author{
Lucila Ishitani \\ Pontifícia Universidade Católica de Minas Gerais \\ Unidade São Gabriel, Belo Horizonte, MG - Brasil \\ lucila@pucminas.br
}

Resumo Modernas tecnologias e padrões de telecomunicação para a computação móvel tornam cada dia mais viável o m-learning. Devido aos diversos problemas geográficos, limitações das soluções Web e expansão do uso de tecnologias móveis, foi proposta a ferramenta m-tutorial, que auxilia professores na elaboração de materiais educacionais para dispositivo móveis, mais especificamente tutoriais voltados para o m-learning. Para demonstrar a aplicabilidade do m-tutorial, são apresentados dois tutoriais desenvolvidos com os recursos disponíveis na ferramenta. Para implementação do mtutorial, foi escolhido o Java ME, por sua portabilidade, por ser de fácil aprendizado, por ser gratuito e por ser projetado para dispositivos com memória, vídeo e poder de processamento limitado. Também foram avaliados APIs, bibliotecas e frameworks para Java ME. Dentre as opções existentes, foram escolhidas as classes da biblioteca Lwuit em conjunto com a tecnologia Web Service. Comparado a outras ferramentas de autoria para desenvolvimento de objetos de aprendizagem para dispositivos móveis, o m-tutorial se destaca porque oferece facilidades na criação de objetos de aprendizagem do tipo tutorial para m-learning, em modo off-line. Os tutoriais podem conter textos, imagens ou vídeos. Seu conteúdo é armazenado internamente em uma classe RMS e enviado para um servidor web quando houver uma conexão estável com a Internet.

Palavras-Chave: Ferramenta de autoria, m-learning, objetos de aprendizagem.

\begin{abstract}
Modern technology and telecommunications standards are developed for mobile computing and $m$ learning. Considering this situation, we proposed a tool - m-tutorial - that assists teachers in preparing interactive educational materials, more specifically, learning objects for m-learning. It aims to build tutorials in a short time and with minimal effort using the existing infrastructure and connectivity for mobile devices. For demonstrating the applicability of the m-tutorial we present two tutorials developed with available resources in the tool. Among the evaluated platforms, Java ME was chosen for its portability, and for being easy to learn, free and designed for limited memory and video processing devices. APIs, libraries and frameworks for Java ME were also evaluated. Among them, we chose to use the LWUIT library classes in conjunction with the Web Service technology. Compared with other mobile applications, m-tutorial stands out because it provides facilities for creating m-learning objects of the type tutorial, in offline mode. Its content is stored internally in a RMS class and sent to a web server when there is a stable connection to the Internet. The tutorials are prepared and may contain text, images or videos.
\end{abstract}

Keywords: authoring tool, m-learning, learning object 


\section{Introdução}

O Brasil tem vivenciado um crescimento considerável na oferta de cursos por meio da Educação a Distância (EAD). Esses cursos favorecem pessoas que têm dificuldades para frequentar cursos tradicionais que exigem presença em horário e locais determinados para sua realização [1]. Professores e alunos não mais necessitam estar no mesmo ambiente e alunos não precisam sacrificar horário de trabalho. Dadas a crescente dificuldade de reunião e locomoção de profissionais e a crescente necessidade de sua capacitação, torna-se necessário criar mecanismos que facilitem o aprendizado contínuo a distância [1].

Dentre as opções de oferta de EAD, há que se considerar o fato de que milhões de pessoas em todo o mundo já se comunicam por meio de tecnologias móveis. Dados da Agência Nacional de Telecomunicações (Anatel) indicam que o Brasil terminou o mês de outubro de $2010 \mathrm{com}$ 189,5 milhões de celulares e uma densidade de 90,6 celular/100 hab. O telefone celular continua com uma tendência de crescimento de uso e já caminha para a universalização nos domicílios brasileiros, chegando a $82 \%$ dos lares em áreas urbanas e $78 \%$ no total do país [2].

Devido a esse contexto, muitos ambientes virtuais de aprendizagem estão sendo adaptados para execução e visualização em telefones celulares [3]. Materiais educacionais digitais, ou Objetos de Aprendizagem (OA), também estão sendo adaptados a este novo cenário.

Entretanto, é importante destacar que, se há professores que sabem desenvolver materiais educacionais digitais, nem todos possuem o conhecimento necessário para desenvolvê-los [4].

Este artigo tem por motivação o suporte ao uso de telefones celulares na aprendizagem móvel, ou m-learning. Com a ferramenta m-tutorial proposta neste trabalho, é possível desenvolver objetos de aprendizagem do tipo tutorial para dispositivos móveis de forma rápida, em qualquer lugar e a qualquer momento, sem precisar de conectividade. Para especificação dessa ferramenta, considerou-se tutorial como um tipo de software educacional que segue "o padrão de ensino da sala de aula tradicional onde o conteúdo é previamente organizado numa estrutura definida pelo professor e o aluno seleciona dentre as diversas opções disponíveis o conteúdo que deseja estudar" [5].

Para validação da ferramenta m-tutorial foram elaborados dois tutoriais como estudo de caso. Esse estudo de caso permitiu comprovar que professores estão dispostos a desenvolver materiais educacionais para m-learning, se houver suporte como o que a ferramenta m-tutorial oferece.

Na Seção 2 deste artigo, são apresentados conceitos e características do m-learning e de objetos de aprendizagem móveis. A Seção 3 discute conceitos básicos sobre APIs e bibliotecas existentes para dispositivos móveis além de mostrar as especificações de algumas APIs. Na Seção 4 são apresentados trabalhos relacionados. Na Seção 5 é apresentada a especificação da ferramenta mtutorial. Na Seção 6 é apresentado um estudo de caso que foi utilizado para validação da ferramenta. Na Seção 7 é apresentado um comparativo entre o m-tutorial e duas outras ferramentas de autoria para dispositivos móveis. Na Seção 8 são trazidas as considerações finais, contribuições e sugestões de trabalhos futuros.

\section{Mobile Learning}

De acordo com Kinshuk et al. [6], "m-learning é definida como a capacidade do uso de dispositivos portáteis para acesso a recursos de aprendizagem". Cabe destacar que a aprendizagem móvel é entendida como mobile $e$ learning e sua história e evolução é uma continuação da aprendizagem convencional.

O m-learning pode oferecer vantagens significativas para as instituições de ensino, tais como: baixos custos de oferta, flexibilidade de acesso virtual aos materiais de informação, possibilidade de aprendizagem coletiva virtual. Os estudos realizados sobre m-learning ainda são recentes. Cada usuário emprega seu dispositivo móvel de uma forma diferente. Por exemplo, adolescentes, frequentemente, usam SMS para se comunicar, enquanto profissionais estão mais propensos a utilizar o e-mail [8].

Uma das características de aprendizagem móvel é que ela utiliza dispositivos móveis [9], que são:

- Usados em qualquer lugar;

- Considerados de uso pessoal;

- Mais baratos que computadores pessoais e mais fáceis de usar;

- Utilizados em diversas configurações.

\subsection{Objetos de aprendizagem móveis}

Para Castillo e Ayala [10], um objeto de aprendizagem móvel é uma entidade de informação digital, interativo, adaptável e reusável nos diferentes contextos, projetado para ser usado em um ambiente de aprendizagem móvel, capaz de suportar aproximações e perspectivas de aprendizagem em diferentes interações. Eles acreditam que objetos de aprendizagem móveis não devem ser ape- 
nas uma extensão simples de objetos de aprendizagem, mas uma adaptação às limitações do formato dos dispositivos móveis.

Para Brown, Collins e Duguid [11] um objeto de aprendizagem móvel deve considerar duas perspectivas de interação, de acordo com a metodologia de aprendizagem:

- $\quad$ Permitir que o usuário interaja com a aprendizagem em qualquer lugar e a qualquer hora;

- Simular situação de aprendizagem a fim de permitir que o aluno participe de uma atividade real a qualquer hora e em qualquer lugar.

\section{APIs e Bibliotecas}

Esta seção fornece uma visão geral de APIs e das principais bibliotecas existentes para a construção de aplicações utilizando dispositivos móveis. A Subseção 3.1 apresenta as principais APIs disponíveis para MIDP utilizadas na criação do m-tutorial. A Subseção 3.2 apresenta as principais bibliotecas baseadas em Canvas.

\subsection{APIs}

A tecnologia Java possui uma comunidade Java Community Process (JCP) conhecida por uma organização internacional de desenvolvedores Java, cujo objetivo é o de desenvolver e rever especificações e implementações. A JCP envolve o uso de Java Specification Requests (JSRs), documentos formais que descrevem as especificações propostas e tecnologias da plataforma Java [12].

Um conjunto de rotinas e padrões são estabelecidos e conhecidos por Application Programming Interface (API). As APIs oferecem recursos diversos. São projetadas para rodar em qualquer máquina virtual baseada em Java ME. Algumas das APIs opcionais disponíveis para MIDP são citadas por Johnson [13]. Todas as APIs listadas nesta seção foram utilizadas em algum momento na criação do m-tutorial e foram selecionadas pela facilidade na utilização:

JSR $139\left(\right.$ CLDC $\left.^{1}\right)$ - Define o conjunto básico de interfaces de programação de aplicativo e máquina virtual para dispositivos com recursos limitados;

JSR 118 (Mobile Information Device Profile) - É uma extensão da API de base fornecida pela configuração CLDC. Permite escrever aplicativos para download e serviços de rede;

1

Connected Limited Device Configuration
JSR 234 (Advanced Mobile Media API) - Define uma API com funcionalidades multimídias avançadas. Ela permite acesso a recursos multimídias mais sofisticados como suporte avançado de câmera, áudio, rádio e processamento de imagem;

JSR 226 (Scalable vector graphics) - Define uma API para desenhar gráficos bidimensionais baseados em gráficos vetoriais (redimensionáveis sem perda de qualidade);

JSR 184 (3D-graphics) - Define uma API leve para gráficos 3D interativos. A API é muito importante para criação de interfaces ricas e desenvolvimento de jogos;

JSR 135 (MultiMedia) - Suporta aplicações multimídia em dispositivos habilitados para Java ME. Ele foi projetado para funcionar com qualquer protocolo e formato, por exemplo, MP3, MIDI, ou MPEG-4;

JSR 75 (File Connection) - Permite acesso e armazenamento em arquivos e acesso a programas nativos do dispositivo;

JSR 82 (Bluetooth) - Permite o desenvolvimento de aplicações para acesso a rede, dependendo da disponibilidade da tecnologia Bluetooth no dispositivo;

JSR 172 (Web Service) - Define um pacote opcional que permite o acesso padrão do Java ME para Web Service.

RMS (Record Management System) - é a solução do J2ME para a criação e manipulação de registros oferecidos pelo Mobile Information Device Profile (MIDP) para persistência de dados. Localizado no pacote javax.microedition.rms, é composto pela classe RecordStore, conhecida como armazém de dados.

\subsection{Bibliotecas}

Bibliotecas são basicamentes arquivos contendo código reutilizável que normalmente pode ser compartilhado entre vários aplicativos. Segundo Horstmann [14], uma biblioteca é um conjunto de objetos e funções que foca na resolução de um problema particular ou uma área específica de desenvolvimento de aplicações, como por exemplo, acessar banco de dados. Utilizando bibliotecas, o desenvolvedor não precisa escrever o mesmo código várias vezes. 


\begin{tabular}{|c|l|c|}
\hline \multicolumn{3}{|c|}{ Principais bibliotecas de Interface Gráfica da Plataforma Java ME } \\
\hline Nome & \multicolumn{1}{|c|}{ Comentários } & Licença \\
\hline Lwuit & $\begin{array}{l}\text { Cria aplicativos com designs mais sofistica- } \\
\text { dos. }\end{array}$ & GPLv2 \\
\hline LwVCL & $\begin{array}{l}\text { Projetada para funcionar em qualquer } \\
\text { plataforma. }\end{array}$ & GPL \\
\hline J2ME & $\begin{array}{l}\text { Visa a auxiliar o programador a lidar com } \\
\text { vários aspectos da interface gráfica na } \\
\text { criação de aplicações para dispositivos de } \\
\text { pequeno porte. }\end{array}$ & GPL \\
\hline J4me & $\begin{array}{l}\text { É uma biblioteca de código aberto. Desen- } \\
\text { volve aplicações mais rápidas, produtivas e } \\
\text { bonitas. }\end{array}$ & $\begin{array}{c}\text { Apache } \\
\text { License 2.0 }\end{array}$ \\
\hline MWT & $\begin{array}{l}\text { Construção de Uls otimizados para celula- } \\
\text { res. }\end{array}$ & LGPL \\
\hline
\end{tabular}

Quadro 1: Principais Bibliotecas de Interface Gráfica da Plataforma Java ME

Do levantamento feito nas bibliotecas listadas, foi decidido utilizar a biblioteca Lwuit por sua facilidade de aprendizado, por ser gratuita e de boa portabilidade.

\subsubsection{Biblioteca Lwuit}

Lightweight UI Toolkit (Lwuit) é uma biblioteca que permite criar interfaces gráficas do usuário para dispositivos móveis ou quaisquer outros dispositivos que suportem o perfil MIDP do Java ME. O Lwuit traz diversos recursos capazes de tornar a interface dos aplicativos em Java ME mais agradável, bonita e simples de usar. Suas principais vantagens são [15]:

- Arquitetura MVC e Multi-plataforma;

- Diversidade de layouts e fontes;

- Diversidade de estilos e temas;

- Suporte a touch screen;

- Suporte a animações e transições;

\section{Trabalhos Relacionados}

Projetos usando dispositivos móveis na educação são divulgados há mais de dez anos. Segundo Houser, Thornton e Kluge [16] projetos educacionais são criados inicialmente nas universidades, escolas e programas de treinamento corporativo de ensino a distância. Esses projetos utilizam celulares como parte de um programa de educação que combina web e dispositivos móveis. Eles mostram que a combinação única de recursos em dispositivos torna-os valiosas ferramentas educacionais, pela facilidade de locomoção e por pessoas carregarem constantemente seus aparelhos celulares. Isso se tornou possível, devido ao surgimento de novas tecnologias que proporcionam uma fácil integração entre as duas plataformas, web e móvel.
Soloway et al. [17] desenvolveram vários programas educacionais para PDAs. Esses programas incluem o jogo de perguntas e respostas Bubble Blasters ${ }^{1}$, simulação de ciência Cooties ${ }^{2}$ e o editor de mapa conceitual PiCo$\mathrm{Map}^{3}$.

Fuks et al. [18] e Fillipo et al. [19] apresentam o AulaNetM, que é uma adaptação do software para $e$ learning AulaNet, para dispositivos móveis. O AulaNetM começou a ser desenvolvido em 2004, para utilização de fóruns de discussão assíncrona em PDAs. O interessante desse trabalho é ter mostrado as dificuldades que a mobilidade trouxe na adaptação do sistema para versão móvel.

De forma mais genérica, Bartholo, Amaral e Cagnin [20] apresentaram o modelo M-AVA, que tem por objetivo apoiar a adaptação de AVAs existentes para o contexto de m-learning. Contudo, por não ser objetivo do trabalho realizado, as autoras não discutem o desenvolvimento de objetos de aprendizagem.

Baseados em projetos de sala de aula, Hadzilacos e Tryfona [21] apresentaram a construção de uma representação multimídia de um sítio arqueológico para aula de história conhecido por Acrópolis. O objetivo do aprendizado consiste em compreender o período histórico por meio da visualização dos edifícios e leitura dos artefatos, obtendo informações dos museus e vista panorâmica dos locais, fazendo uma descrição verbal dos monumentos arquitetônicos. Os alunos podem visitar o sítio arqueológico e o museu várias vezes. Trabalhando em dupla podem solicitar orientações a seu professor a qualquer hora. Caso o aluno não reconheça um determinado objeto, pode fazer uma pesquisa na base de dados de seu dispositivo. $\mathrm{O}$ interessante desse projeto foi a possibilidade de utilização de aprendizagem coletiva entre professores e alunos.

Santos et al. [22] propuseram um framework para a construção de aplicações educacionais para dispositivos móveis, com recursos de realidade virtual, chamado Virtual Training for Mobile Device (VirTraM), que foi utilizado na criação de um museu virtual. O protótipo desenvolvido demonstra interatividade, usabilidade e portabilidade, além de permitir uma experimentação da integração das tecnologias de realidade virtual e computação móvel. Este framework falha por não possuir uma interação com a web e não utilizar recursos tecnológicos como Global Positioning System (GPS), Radio Frequency Identification (RFID) e wireless, tecnologias necessárias para permitir a localização automática no passeio ao museu.

Para finalizar, na literatura foram encontradas duas

\footnotetext{
${ }^{1}$ http://download.cnet.com/Bubble-Blasters/3000-18495\4-16840.html

${ }^{2}$ http://www.goknow.com/Products/Cooties/

${ }^{3}$ http://www.goknow.com/Products/PiCoMap/
} 
ferramentas Mobile Author Learning ${ }^{1}$ e MobileSiteGalo$\mathrm{re}^{2}$ para criação de OAs para m-learning disponíveis na web. O Mobile Author permite projetar, testar e publicar conteúdo móvel para a utilização em telefones celulares e PDAs. Essa ferramenta executa OAs feitos para qualquer aparelho celular a partir do desktop. Já o MobiSiteGalore é o pioneiro entre as ferramentas construtoras de páginas web para dispositivos móveis com facilidade. O interessante dessa ferramenta é que ela funciona em qualquer telefone móvel, gratuitamente, sem anúncios. Não requer conhecimentos técnicos e nem software específico para instalá-la, apenas um navegador da web no celular. A deficiência dessa ferramenta está em não permitir a criação de objetos de aprendizagem em modo off-line. De todas as ferramentas e frameworks avaliados, o m-tutorial se diferencia pela possibilidade de criar objetos de aprendizagem em modo off-line e enviá-los a um servidor quando possuir uma conectividade estável com a Internet.

\section{Especificação do m-tutorial}

O desenvolvimento de uma ferramenta de autoria demanda que desenvolvedores tenham a possibilidade de examinar os requisitos e estudar as funcionalidades a partir de diversos diagramas.

Nesta seção são apresentados os modelos UML (Diagrama de caso de uso, Diagrama de classes, Diagrama de componentes e Diagrama de implantação) utilizados na elaboração do m-tutorial.

\subsection{Diagrama de caso de uso}

O Diagrama de caso de uso descreve os requisitos funcionais da ferramenta. Ele apresenta a troca de informações entre atores (usuários) e as ações que serão executadas no m-tutorial. O Quadro 2 descreve as responsabilidades atribuídas aos atores.

\begin{tabular}{|l|l|}
\hline \multicolumn{2}{|c|}{ Atores } \\
\hline Professor & $\begin{array}{l}\text { Este ator interage por meio de uma interface gráfi- } \\
\text { ca. Ele consegue criar novos objetos de aprendiza- } \\
\text { gem, por conta própria ou seguindo um assistente. }\end{array}$ \\
\hline Aluno & $\begin{array}{l}\text { Este ator visualiza conteúdo e faz download de } \\
\text { arquivos. Ele interage com os OAs criados. }\end{array}$ \\
\hline Administrador & $\begin{array}{l}\text { Este ator interage por meio de uma página web. Ele } \\
\text { atualiza os parâmetros do servidor e mantém novos } \\
\text { usuários. }\end{array}$ \\
\hline
\end{tabular}

Quadro 2: Responsabilidades atribuídas aos atores
De acordo com a Figura 1, as funcionalidades da ferramenta m-tutorial são:

Manipular objetos de aprendizagem: Este requisito propiciará a criação, a alteração e a exclusão dos objetos de aprendizagem. Esses objetos podem conter textos, imagens ou vídeos.

Manter usuário: o requisito permite manter o perfil de acesso dos usuários e determinar a quantidade de acessos dos usuários.

Visualizar OA: Por este requisito é possível visualizar o objeto de aprendizagem na tela do celular.

Manter formato: Este requisito determina o tipo de formato utilizado na importação e exportação de arquivos.

Realizar upload: $\mathrm{O}$ requisito citado possibilita ao professor realizar o upload do objeto de aprendizagem criado.

Realizar download: $O$ requisito citado possibilita ao aluno fazer o download do objeto de aprendizagem em um repositório de dados.

Manter metadados: Através da utilização deste requisito é possível a criação, edição, exclusão e pesquisa por metadados.

Utilizar wizard: Este requisito permitirá aos usuários do m-tutorial criar objetos de aprendizagem de forma automatizada, seguindo e cumprindo etapas durante a construção do mesmo. É semelhante ao primeiro caso de uso ("Manipular objetos de aprendizagem"), com a diferença de ser realizado por meio de um wizard.

Manter parâmetro: Este requisito permite ao administrador atualizar parâmetros utilizados pela ferramenta.

Na Figura 1 podemos observar o diagrama de caso de uso da ferramenta m-tutorial.

\footnotetext{
${ }^{1}$ http://www.abjade.com

${ }^{2}$ http://www.mobisitegalore.com
} 


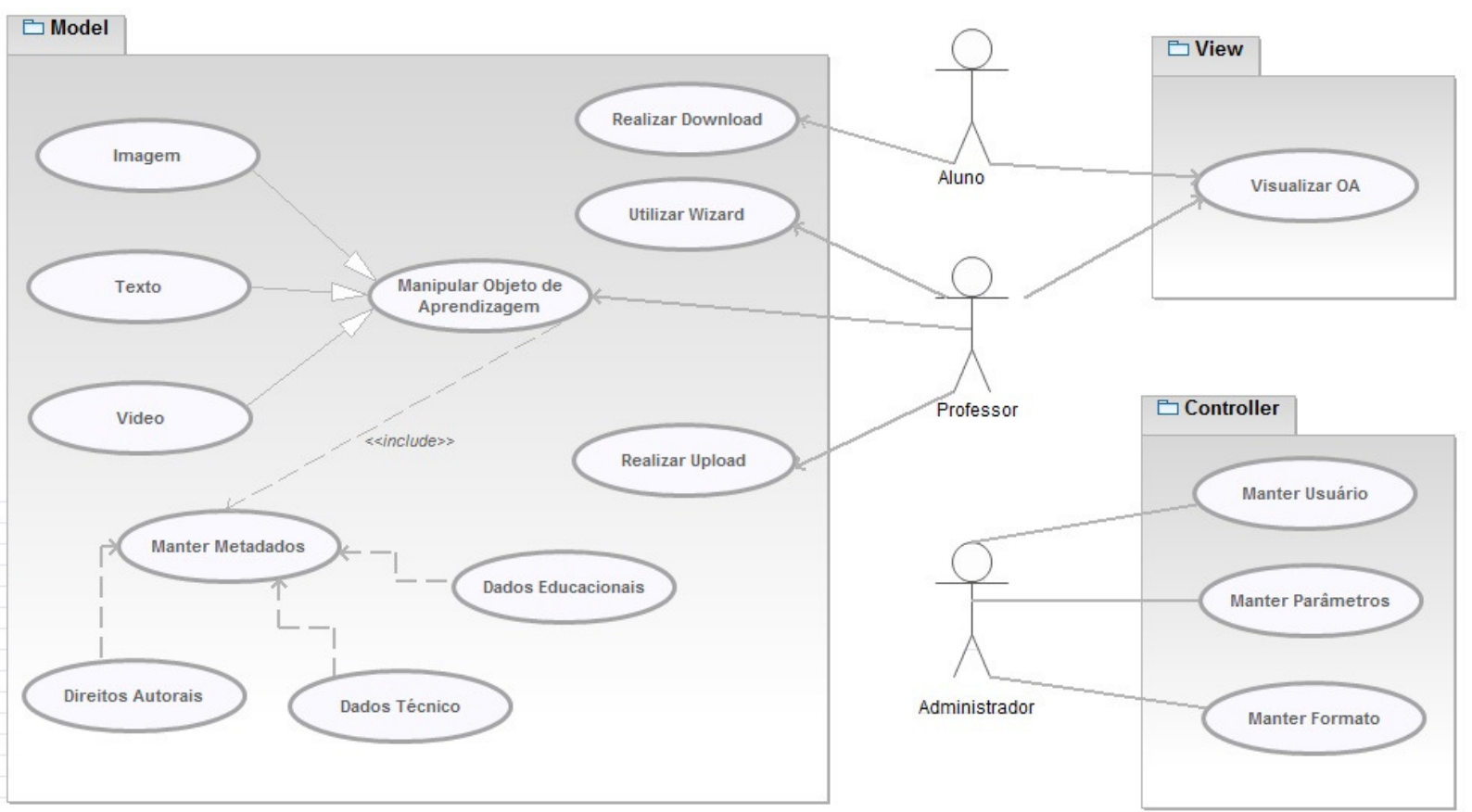

Figura 1: Diagrama de caso de uso do m-tutorial

\subsubsection{Diagrama de classes}

Esta subseção apresenta as principais contribuições da ferramenta, na forma de um diagrama. São listadas classes e métodos de maior relevância do m-tutorial.

Deseja-se com essa ferramenta oferecer a possibilidade das pessoas construírem objetos de aprendizagem do tipo tutorial em qualquer dispositivo móvel independente do lugar, não importando estar conectado.

O Diagrama de classes da Figura 2 permite a visualização dos objetos que compõem a estrutura da ferramenta m-tutorial. Esse modelo de classes foi validado por um checklist aplicado na fase inicial do ciclo de vida da fer- ramenta.

No pacote View, ações do usuário como cliques ou teclas pressionadas são capturadas. As classes desse pacote estão modeladas de forma que qualquer desenvolvedor consiga reutilizá-las em outras aplicações. A classe principal da ferramenta é chamada de "DispositivoMovel". Por esse objeto é possível navegar em uma lista onde diversos serviços são oferecidos.

No pacote Controller é apresentado o fluxo da aplicação.

Já no pacote Model são apresentadas as classes responsáveis por tudo que a aplicação vai fazer, como armazenamento, manipulação e geração de dados. 


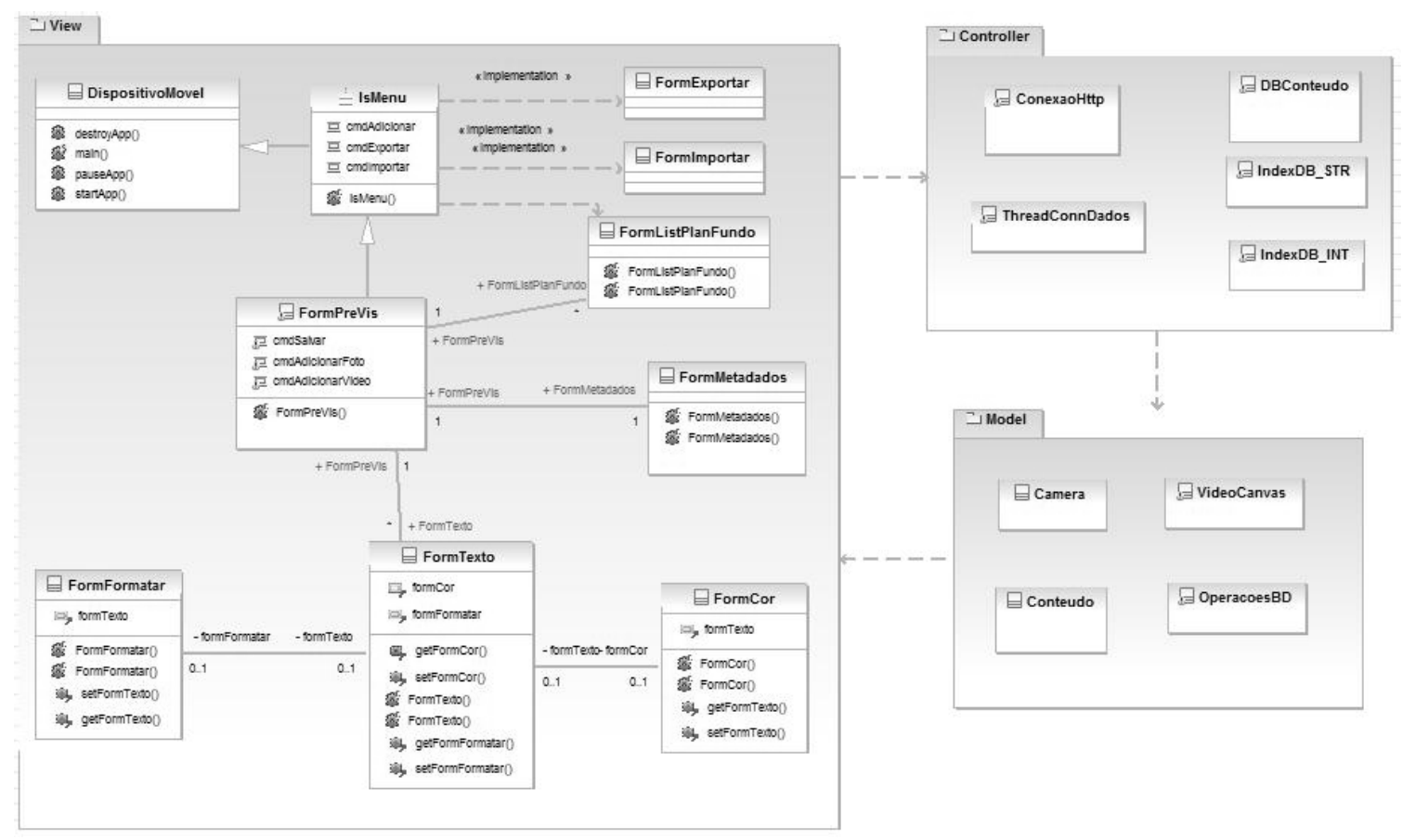

Figura 2: Diagrama de classes do m-tutorial

\subsubsection{Diagrama de componentes}

O Diagrama de componentes da Figura 3 descreve a funcionalidade desempenhada pelos componentes inter- nos e externos da ferramenta. Os componentes internos instanciam as classes concretas com os objetos textos, imagens e vídeos criados. Já os componentes externos pertencem à biblioteca Lwuit.

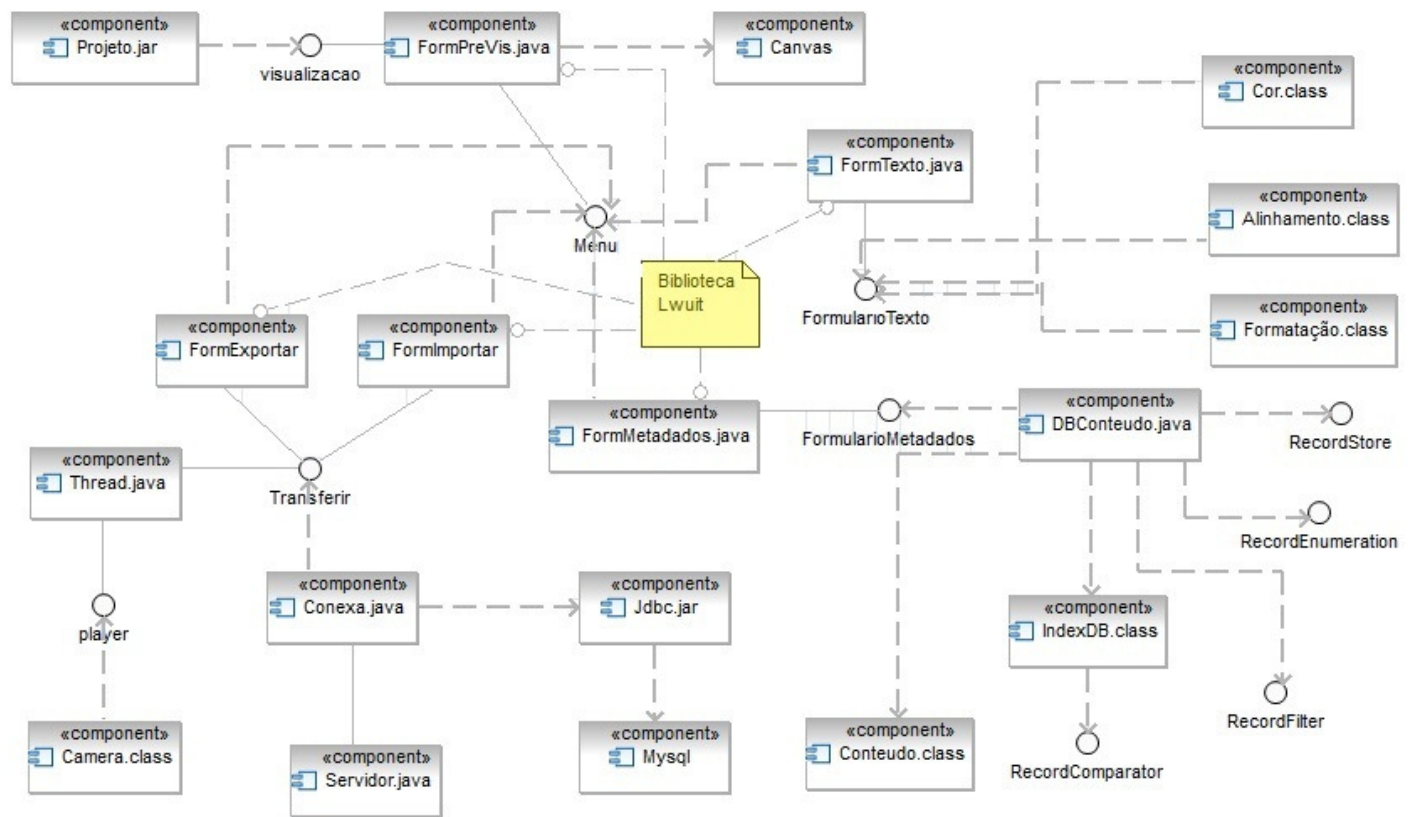

Figura 3: Diagrama de componentes do m-tutorial 
O Diagrama mostra as dependências entre componentes de software. O componente FormPreVis, responsável por listar o conteúdo no formulário de visualização, verifica se existe algum texto ou imagem no formulário de pré-visualização. Caso o formulário esteja vazio, novas imagens e textos serão armazenados para serem prévisualizados. Caso contrário, o formulário deve ser limpo, excluindo ou armazenando os objetos nele contidos.

O componente Camera é o responsável por efetuar as ações com os dados multimídia, como apresentar no display do celular a câmera do aparelho. Define qual a origem dos dados utilizados. Esses dados podem vir da câmera do celular, de um arquivo binário (mp3, mp4, wav), ou de um endereço na web.

Quando o objeto player do componente Camera mudar seu estado para "REALIZADO", é iniciada a captura dos dados gerados pela câmera. Em seguida, uma função informa onde serão visualizados os dados gerados pela câmera do dispositivo.

Já a instância VideoCanvas é a responsável por apresentar o conteúdo recebido pelo display do celular. VideoCanvas é uma extensão de Canvas, isso significa que todas as características que essa classe possui, foram herdadas de Canvas. A principal delas é a possibilidade de desenhar pixel a pixel a tela do dispositivo.

Para a comunicação com o Web Service utilizou-se uma das tecnologias de comunicação conhecida por HTTP: protocolo de transferência de dados construído sobre o protocolo TCP/IP utilizado na web.

Em aplicações que envolvem serviços web, uma requisição HttpConnection deve operar em componente Thread separado, caso contrário a aplicação ficará travada enquanto sua requisição estiver se comunicando com o servidor. Será possível inclusive disparar várias conexões paralelas e, ainda assim, a aplicação terá, ao receber os dados, o controle de qual conexão se trata.
O Componente Conexa é o responsável por todo o processo de comunicação com o servidor, como por exemplo, controle de erros, timeout, leitura e envio de dados entre outros. No final da requisição, esse componente envia para o usuário um aviso de que a requisição terminou.

Já o componente DBConteudo é o responsável no armazenamento dos dados em um RMS. O mecanismo de armazenamento do RMS é implementado como uma coleção de registros onde cada registro é organizado como um array de bytes.

Os componentes apresentados nesta seção poderão auxiliar o desenvolvimento de diversas aplicações para dispositivos móveis

\subsubsection{Diagrama de implantação}

Com intuito de distribuir as responsabilidades de cada parte da aplicação, escolheu-se utilizar o padrão de projeto Model-View-Controller (MVC), por resolver problemas de interação entre usuário e sistemas.

O m-tutorial utiliza protocolos baseados em padrões XML que permitem a geração automática tanto do código cliente quanto do código do servidor. A comunicação distribuída ocorre por meio de mensagens XML, formatadas e encapsuladas segundo o protocolo SOAP e transportadas via HTTP.

A conexão do servidor web com um sistema gerenciador de banco de dados realiza-se por meio de um drive que trata de todo o processo de comunicação.

A Figura 4 ilustra o Diagrama de implantação da ferramenta m-tutorial. Apresenta também como a aplicação cliente pode utilizar-se de periféricos do celular como câmera digital, microfone e, futuramente, impressora. 


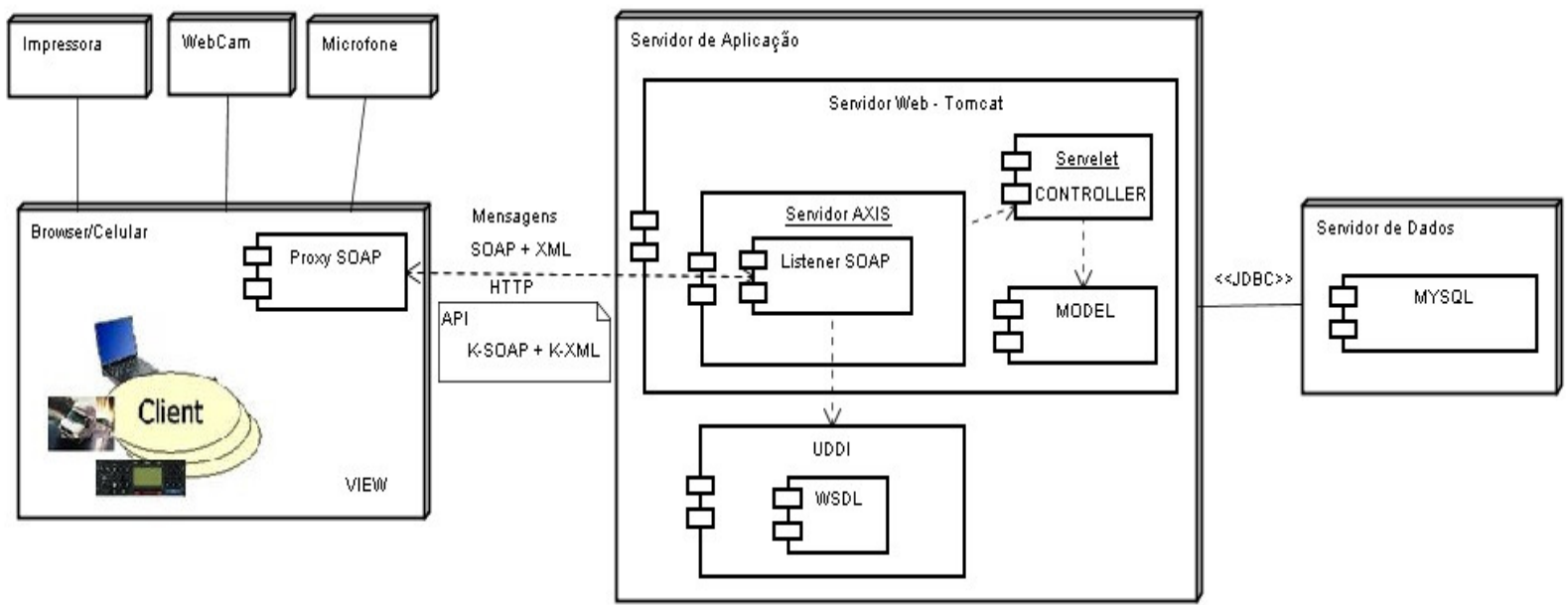

Figura 4: Diagrama de implantação do m-tutorial

Para desenvolvimento desse Web Service utilizou-se o Apache Axis que será executado sobre o apache Tomcat. O Axis basear-se-á em arquivos jws para criar os arquivos de definição WSDL. Para consumir esse Web Service instalado no Apache Axis, usou-se um aplicativo cliente utilizando a tecnologia Java ME, que se conecta ao servidor utilizando as API's KXML e KSOAP. Essas APIs permitem aos dispositivos móveis acessarem Web Services baseados em XML. Esse funcionamento se dá pela codificação, serialização e envio dos métodos e seus argumentos. Esses métodos são recebidos, decodificados e desserializados de maneira transparente pelo servidor.

Das plataformas avaliadas, optou-se por utilizar JME por sua portabilidade, por ser de fácil aprendizado, gratuito, projetado para dispositivos com memória, vídeo e poder de processamento limitado. Segundo Buschmann et al. [23], há atualmente quase 2 bilhões de aparelhos com essa plataforma, imensa comunidade de desenvolvedores e seu kit de desenvolvimento esta disponível para Windows, Linux e Mac OS X.

Para desenvolver a aplicação deste projeto foi utilizada a IDE NetBeans 6.7 Full, a qual possui o plug-in Mobility Pack usado no desenvolvimento visual de aplicações para celular.

\section{Desenvolvimento de Objetos de A- prendizagem utilizando a ferramen- ta m-tutorial}

A ferramenta implementada foi desenvolvida para criar objeto de aprendizagem de forma dinâmica e interativa, propiciando a criação de tutoriais a qualquer hora e lugar. $\mathrm{O}$ aplicativo é composto por formulários intuitivos que seguem uma ordem cronológica para o desenvolvimento de tutoriais. Toda a interação é feita com a utilização das teclas do telefone celular.

Ao iniciar o protótipo, o usuário é direcionado para o formulário principal da ferramenta. Nessa tela será visualizada uma lista com as principais funcionalidades do protótipo, conforme ilustra a Figura 5.

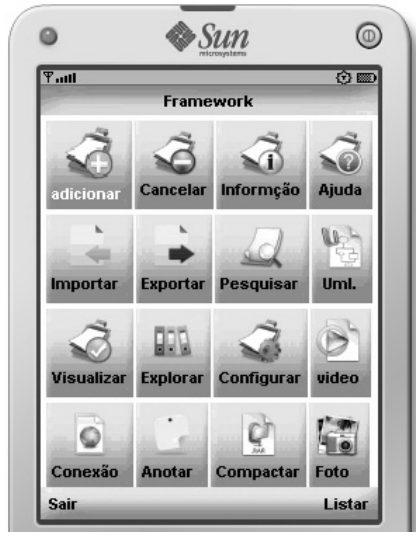

Figura 5: Tela inicial da ferramenta m-tutorial

Ao escolher a opção criar um novo tutorial pela opção wizard, o usuário é encaminhado a uma tela de instruções que oferece orientação para escolha de um plano de fundo. Em seguida é solicitado ao usuário a inserção de um novo texto ou imagem. Caso seja escolhido adicionar texto, um novo formulário é exibido, oferecendo a inserção e edição do texto, com as opções: fonte, estilo, tamanho, sublinhado, cor do texto e alinhamento. Mas se a escolha for pela inserção de uma imagem, o usuário poderá selecionar a imagem desejada em uma lista conti- 
da em seu aparelho ou, se o dispositivo possuir câmera, uma nova foto poderá ser tirada e adicionada em seu tutorial.

Após a formatação do texto ou imagem, tendo finalizado o tutorial, o usuário salvará o objeto de aprendizagem. Para finalizar o processo, deverá preencher um formulário de metadados informando os campos: título, autor, área, nível, público, idioma e versão.

Com os campos do formulário de metadados preenchidos, o conteúdo dos campos é adicionado em uma

a)

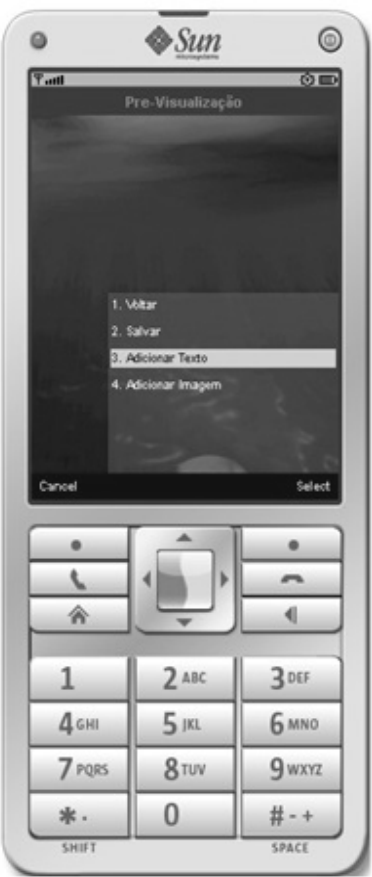

b)

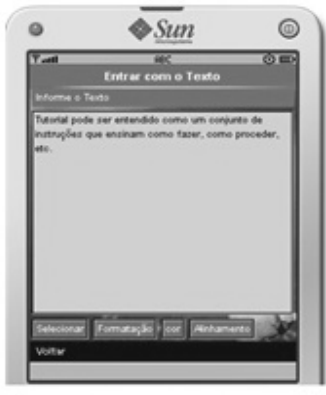

d)

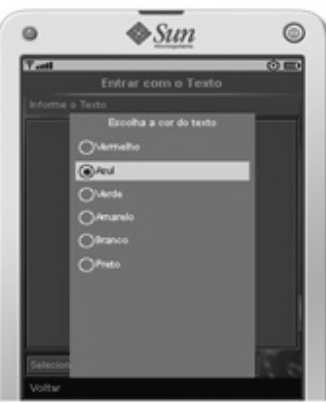

lista armazenada na memória do dispositivo móvel. Se o RMS estiver vazio (primeiro acesso), a lista inicia sem dados. Nos demais acessos serão apresentados os registros armazenados no RMS.

Após apresentada a lista principal, o usuário terá a opção de incluir um novo tutorial por meio da opção adicionar, na qual será chamado um novo formulário. A Figura 6 apresenta um exemplo produzido com a menta m-tutorial capturadas a partir do emulador less Toolkit.

c)

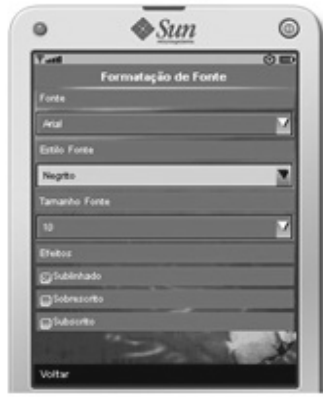

e)

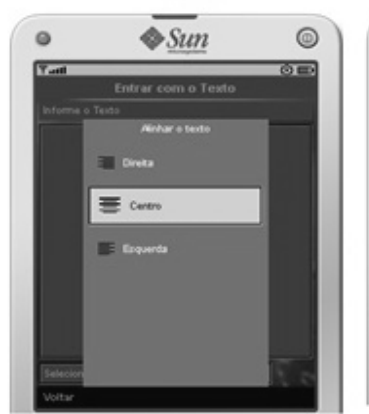

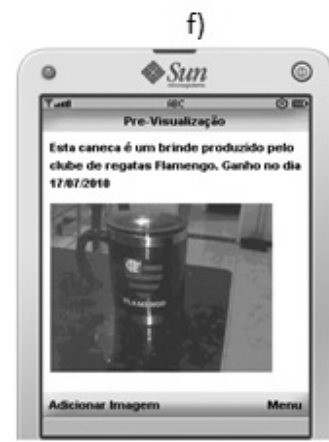

g)

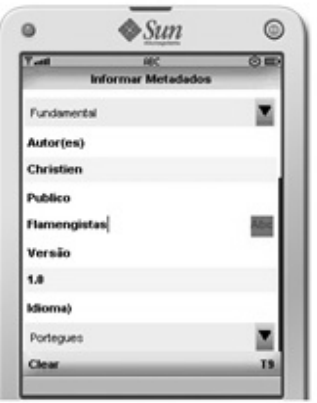

Figura 6: a) Tela de seleção de texto ou imagem. b) Uma visão da inserção do texto. c) Tela de formatação do texto. d) Uma visão da escolha de cor do texto.e) Tela de alinhamento do texto. f) Uma visão final do tutorial. g) Tela de inserção dos metadados.

O produto gerado pelo m-tutorial é um arquivo do tipo HTML, que pode ser visualizado em qualquer dispositivo móvel. O m-tutorial se caracteriza pela possibilidade de criar tutoriais adaptáveis a qualquer tamanho de tela e dispositivo.

\subsection{Testes em Ambiente Real}

Os testes em ambiente real da ferramenta m-tutorial aconteceram em duas etapas. Na primeira etapa, o tutorial foi elaborado utilizando o aparelho celular Vaic F029, cujo sistema operacional é o Symbian OS. A Figura 7 ilustra a criação de tutoriais no aparelho celular, fora do simulador. 


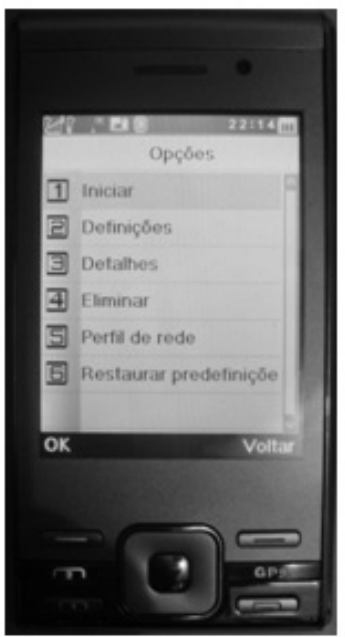

a)

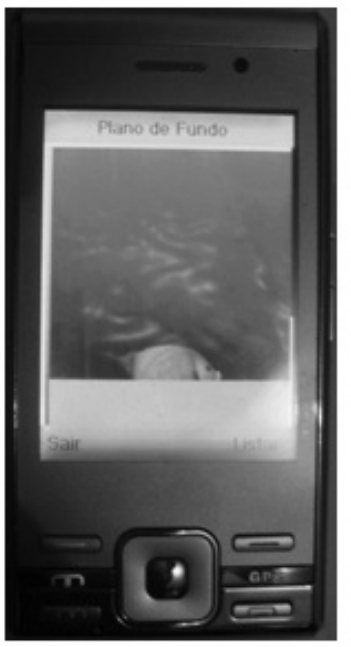

d)

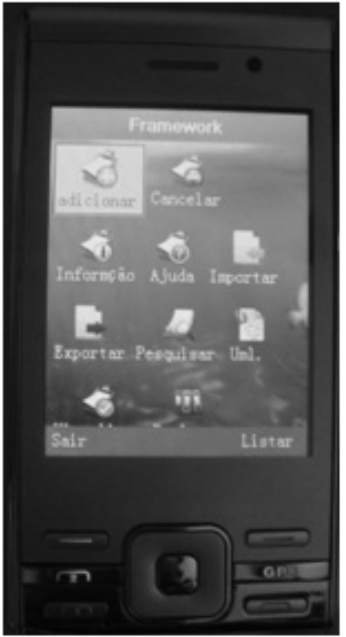

b)

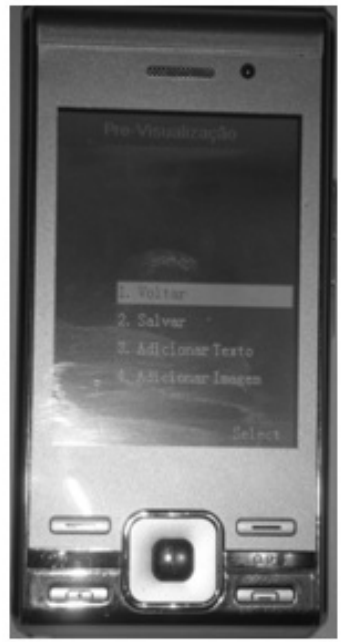

e)

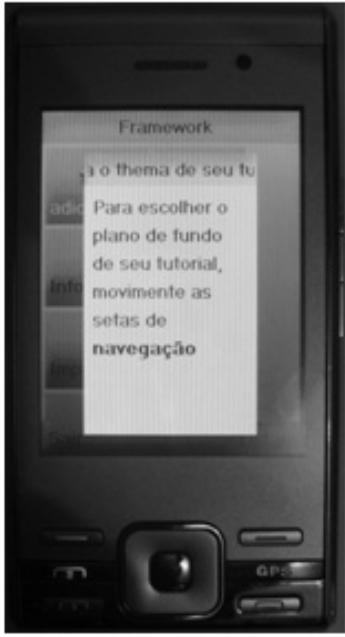

c)

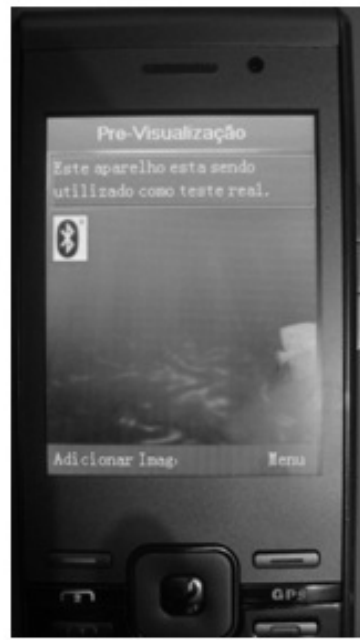

f)

Figura 7: a)

Tela inicializar o m-tutorial b) Tela Principal com a função adicionar objeto de aprendizagem. c) Tela informativa plano de fundo.d) Uma Visão da Escolha de plano de fundo. e) Tela de escolha de texto, imagem ou salvar. f) Uma visão Final do Tutorial.

$\mathrm{Na}$ segunda etapa dos testes, o m-tutorial na web foi avaliado no smartphone Nokia E61. Na Figura 8 é apresentada a página inicial com a funcionalidade de criação de objetos de aprendizagem, podendo escolher a inserção de um plano de fundo, texto, imagem ou vídeo. Também são mostradas as opções de upload, download e pesquisa por objetos de aprendizagem.

\section{Avaliação do m-tutorial}

Com o propósito de fazer uma avaliação da proposta do m-tutorial, foram estabelecidos critérios para a execução de uma comparação entre ferramentas de autoria para m-learning. Os critérios adotados foram: nível de reutilização, quantidade de documentação disponível, usabilidade, modularização, segurança, eficiência e custos.

As ferramentas escolhidas para este comparativo foram as já citadas na seção de Trabalhos Relacionados, por estarem disponíveis na Web, para uso: Mobile Author e mobiSiteGalore. 


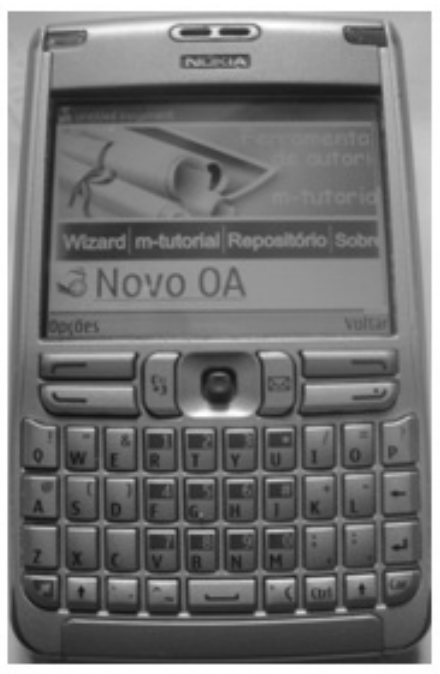

a)

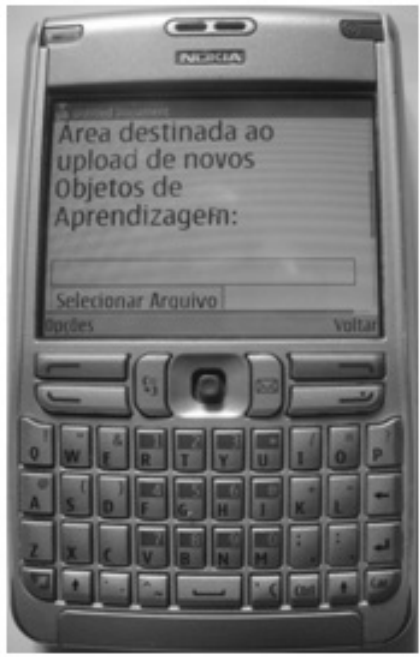

d)

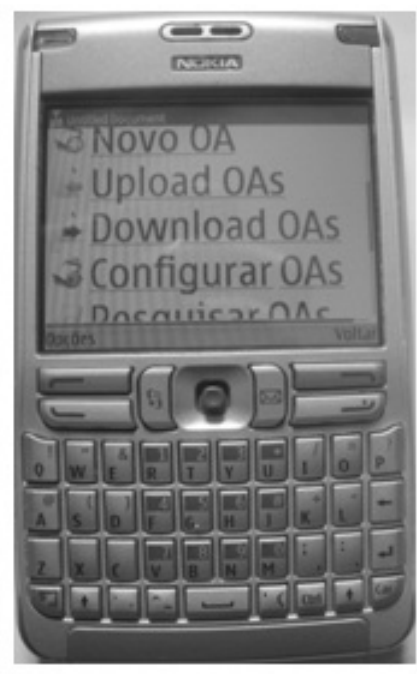

b)

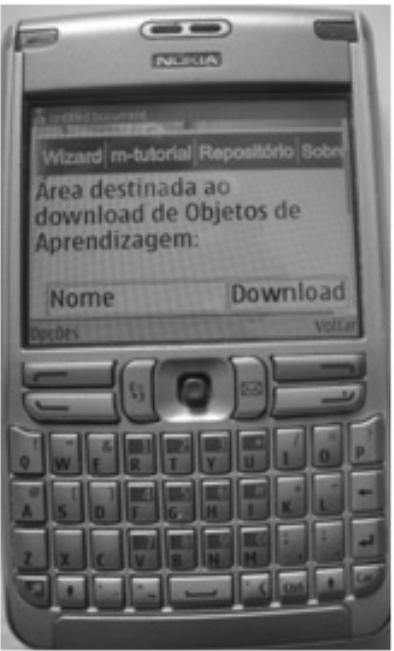

e)

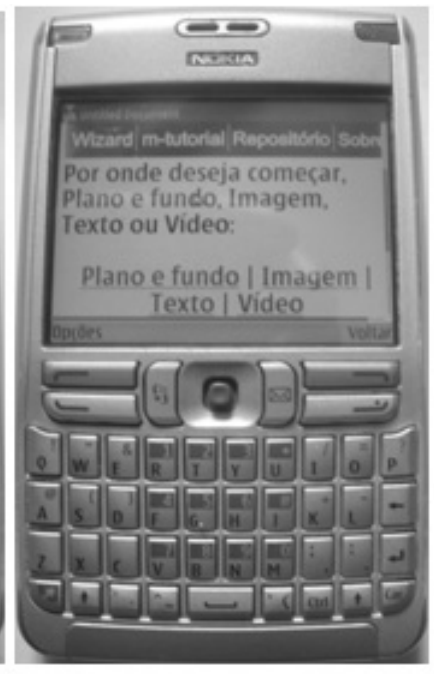

c)

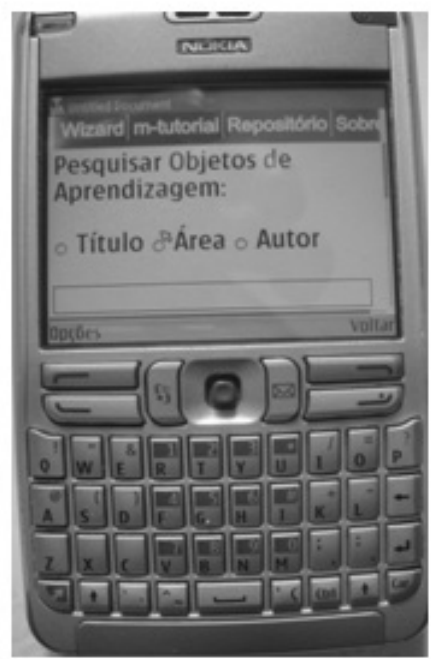

f)

Figura 8: a) Tela inicial da ferramenta m-tutorial. b) Tela inicial da ferramenta m-tutorial. c) Tela de seleção de plano de fundo, texto, imagem ou vídeo. d) Tela de upload de objetos de aprendizagem. e) Tela de download de objetos de aprendizagem. f) Pesquisar objetos de aprendizagem.

Reutilização: No comparativo feito entre as três ferramentas pode-se perceber que o m-tutorial foi elaborado pela instanciação de classes concretas por meio dos objetos textos, imagens e vídeos criados. Isso permite a geração de código seguindo uma arquitetura de referência. Já o Mobile Author e mobiSiteGalore são aplicações proprietárias, suas classes são protegidas, por isso não foi possível avaliar esse item.

Documentação: Uma boa documentação é fundamental para o sucesso de uma ferramenta. Realizou-se um levantamento da documentação oferecida pelas ferramen- tas comparadas. O m-tutorial mais uma vez se destacou. Sua documentação faz parte do protótipo. Embasamento teórico e modelos UML são oferecidos a desenvolvedores de aplicações. As ferramentas mobiSiteGalore e Mobile Author baseiam sua documentação no usuário final. Nenhuma documentação de classes é oferecida. Sua documentação é voltada para manuais de usuário para auxiliálos em alguma tarefa. Não estão interessados em oferecer detalhes técnicos ou administrativos.

Usabilidade: As ferramentas avaliadas são voltadas para criação de objetos de aprendizagem para dispositi- 
vos móveis. A facilidade de uso foi o destaque das ferramentas. Para utilizá-las não é necessário nenhum conhecimento técnico prévio, habilidade de programação ou experiência em web design. Com mobiSiteGalore pode-se escolher um dentre vários templates, que facilitam o desenvolvimento de um site para dispositivo móvel. Uma vez escolhido, é possível customizar completamente as cores, fontes e layout do site. No Mobile Author a criação de OAs é feita com poucos cliques. Já o m-tutorial foi projetado com intuito de possibilitar a construção de OAs no próprio aparelho celular. Foi realizada uma avaliação heurística com dois estudantes do mestrado de Ciência da Computação sendo um da Universidade Federal de Viçosa e outro da PUC Minas e um professor da disciplina de Interface Usuário Máquinas da Universidade Presidente Antônio Carlos. A proposta da avaliação heurística foi inspecionar o protótipo da ferramenta m-tutorial realizada por meio de sessões curtas de avaliação individual. A avaliação gerou algumas sugestões de melhoria, que incluíram a adequação dos objetos de aprendizagem gerados a padrões já existentes.

Modularidade: A modularidade de uma ferramenta se baseia na combinação de componentes. Um módulo, normalmente, utiliza os serviços de outros módulos não sendo, portanto, considerado um sistema independente. Verificou-se que todas as três ferramentas possuem características modulares. O Mobile Author, por exemplo, possibilita adicionar módulos como imagens, som, vídeo, texto, listas numeradas e listas com marcadores. A nova versão do mobiSiteGalore permite incorporar em seu web site vídeos do YouTube, Google Maps, imagens, áudio e arquivos. Já o m-tutorial combina módulos manipuláveis com tarefas específicas como, por exemplo, os objetos imagens, áudio e vídeo.

Segurança: Para que ferramentas sejam consideradas seguras, elas devem ser projetadas de modo a garantir a segurança de quem programa e, principalmente, de quem usa o que foi feito a partir delas. O m-tutorial possui classes de regras de negócio como ponte para a camada de banco de dados e permissões de acesso a código que representam o acesso a um recurso protegido ou a capacidade de executar uma operação protegida. Só código com confiança suficiente pode atender a recursos protegidos do sistema. O mobiSiteGalore foi testado e certificado para ser extremamente confiável. Todos os processos, desenvolvimento e suporte são certificados para serem totalmente compatíveis com as normas de qualidade ISO 9001:2000. Já o Mobile Author em nenhum momento apresenta informações sobre segurança e suas classes são de uso particular, impossibilitando a realização de alguns testes.

Eficiência: Para medir a eficiência das ferramentas levou-se em conta o tempo gasto no processo de criação do objeto de aprendizagem. No mobiSiteGalore, o tempo médio de 54 minutos é o suficiente para criar e visualizar um site Wap em um celular. O objeto de aprendizagem é suportado por qualquer aparelho com um navegador móvel [24]. No m-tutorial, o tempo de criação de um objeto de aprendizagem varia de acordo com a capacidade de armazenamento dos dispositivos móveis e das necessidades do usuário. Os tutoriais são elaborados podendo conter textos, imagens ou vídeos. O tempo médio na criação de um tutorial varia. Um tutorial simples gasta uma média de 8 minutos para ser finalizado. Já o Mobile Author [25] permite criar checklist, questionários, testes e enquetes gastando, na média, 10 minutos, dependendo do tamanho do objeto criado.

Custos: A ferramenta m-tutorial foi desenvolvida por meio da licença "general public license version 2"(GPLv2), no qual a obrigação de restringir a liberdade de outros implica não poder distribuir o programa. Uma versão do m-tutorial se encontra disponível em www.christienrachid.com.br/mtutorial. A ferramenta mobiSiteGalore não oferece uma versão Premium ou paga. Todos os recursos liberados até agora são de uso gratuito. Porém, nenhum código ou API para interagir com a ferramenta estão disponíveis. Já a versão gratuita do Mobile Author inclui apenas ferramentas de criação de conteúdo. Para adquirir a versão completa é necessário o pagamento de uma licença comercial.

Mobilidade da Aprendizagem: Todas as ferramentas comparadas são excelentes para utilização da mobilidade na aprendizagem. $\mathrm{O}$ m-tutorial proporciona ao usuário a criação de objeto de aprendizagem do tipo tutorial, que pode ser utilizado em modo off-line a qualquer hora em qualquer dispositivo móvel. Seu conteúdo é armazenado internamente em uma classe RMS e enviada para um servidor web quando houver uma conexão estável. No Mobile Author, objetos de aprendizagem com recursos multimídia são criados a partir de um PC, podendo ser executados em qualquer tipo de aparelho móvel. Já mobiSiteGalore é uma aplicação baseada em Web Service. Atua na construção de objetos de aprendizagem do tipo $w e b$ site. Esse objeto de aprendizado pode ser criado a partir de um celular, com a objeção de que o desenvolvedor deve sempre estar conectado para conseguir criar um objeto.

Aprendizagem Colaborativa: Aprendizagem colaborativa é a situação na qual duas ou mais pessoas aprendem ou tentam aprender, conjuntamente, alguma coisa [26]. Das três ferramentas avaliadas, nenhuma delas se enquadra nesse contexto de aprendizagem.

Recursos dos Dispositivos Móveis: Este item validou se alguma das ferramentas comparadas utiliza recursos oferecidos pelos aparelhos celulares, como filmar, gravar áudio, tirar fotos, transferir dados por bluetooth entre 
outros. O m-tutorial utiliza um método capaz de manipular a câmera dos dispositivos móveis, possibilitando incluir recursos como imagem e vídeo em seus tutoriais. Já as outras duas ferramentas também utilizam de recursos multimídias, porém não possibilitam utilizar recursos do celular na criação de objeto de aprendizagem.

\section{Conclusão}

Para estimular o uso de objetos de aprendizagem voltados para $m$-learning, é necessário fornecer recursos aos professores para o desenvolvimento desses objetos. Neste trabalho, foi apresentada a ferramenta m-tutorial, que possui as seguintes características: objetos de aprendizagem são de fácil publicação e podem ser criados em um aparelho celular, mesmo estando em modo off-line.

Para melhor visualização do que pode ser construído com a ferramenta de autoria, foram apresentados dois tutoriais construídos a partir das funcionalidades do mtutorial. A facilidade de desenvolvimento está em interfaces intuitivas e bem documentadas de forma que qualquer usuário consiga interagir e criar OAs de forma rápida.

Este trabalho envolveu o desenvolvimento de aplicações para dispositivos móveis utilizando tecnologias como Web Service, XML, Java ME, RUP, UML. Diversas tecnologias foram avaliadas, com um foco em ferramentas para m-learning. Esse estudo propiciou a escolha da biblioteca Lwuit dentre os diversos existentes.

Como os dispositivos móveis estão se transformando em importantes ferramentas para educação e treinamento, o m-tutorial foi desenvolvido seguindo as técnicas de engenharia de software, para que a especificação da ferramenta possa ser reaproveitada. Suas classes foram especificadas e implementadas seguindo características que um framework deve possuir para ser reusável, extensível, seguro, eficiente e completo.

Para validação da ferramenta, foi estabelecido um conjunto de critérios de avaliação e foi realizada uma comparação entre o m-tutorial e outras duas ferramentas de autoria de OAs para m-learning. Os critérios considerados no comparativo podem contribuir para os desenvolvedores de novos aplicativos para desenvolvimento de OAs para dispositivos móveis.

A partir dos resultados apresentados, pode-se perceber que o m-tutorial, apesar de oferecer poucas funcionalidades, permite aos professores a elaboração de OAs, sem a utilização de uma conectividade existente para dispositivos móveis.

Como trabalhos futuros, propomos a construção de componentes adicionais, como um componente que permita criação de páginas web para dispositivos móveis, feitas no próprio aparelho.
Como o m-tutorial tem sua arquitetura baseada no Lwuit, seria interessante adaptar as suas classes para uso na TV digital, oferecendo aos professores uma forma de criar objetos de aprendizagem do tipo tutorial pelo celular, para serem visualizados na TV. Suportado no Ginga-J da TV digital brasileira, o Lwuit TV é bastante semelhante ao Lwuit no celular.

Tutoriais criados no formato de áudio ou vídeo são os grandes desafios do m-tutorial. Os recursos oferecidos pelos aparelhos móveis ainda são muito limitados. Arquivos de vídeos ocupam muito espaço e memória para funcionarem sem interrupções. Deseja-se oferecer classes que possibilitem ao conteudista elaborar objetos de aprendizagem em diversas extensões. Um tutorial criado em áudio ou vídeo e armazenado no próprio aparelho poderá ser enviado para um repositório de dados ofertado na web quando possuir conectividade com a Internet.

Além disso, é necessário envolver mais professores e alunos na avaliação da ferramenta e dos objetos de aprendizagem gerados para uma melhor identificação das melhorias que devem ser incorporadas em projetos futuros de ferramentas de autoria de objetos de aprendizagem para m-learning.

Deve-se pensar, ainda, em recursos que apóiem a reutilização de objetos de aprendizagem móveis e a aprendizagem colaborativa.

Espera-se que os resultados deste trabalho possam auxiliar arquitetos de software e usuários conteudistas na construção de novas aplicações para m-learning.

\section{Referências}

[1] L. Pelissoli, W. Loyolla. Aprendizado móvel (mlearning): Dispositivos e cenários. In: Anais do 11 o Congresso Internacional de Educação a Distância. Salvador, BA, BRASIL: [s.n.], 2004.

[2] CETIC.BR. Uso das Tecnologias da Informação e da Comunicação (TIC). 2009. Disponível em: http://www.cetic.br/ Acesso em: 17 de Set. 2009.

[3] F. T. Franciscato, R. D. Medina. Sistema de gerenciamento de objetos de aprendizagem para dispositivos móveis. Novas Tecnologias na Educação, CINTED, UFRGS, RS, BRASIL, v. 7, n. $1,2009$.

[4] LTSC. Draft Standard for Learning Object Metadata. IEEE 1484.12.1-2002. 2002.

[5] L. M. M. Giraffa. Uma odisséia no ciberespaço: O software educacional dos tutoriais aos mundos 
virtuais. Revista Brasileira de Informática na Educação, v. 17, n. 1, 2009

[6] Kinshuk, S. Jarkko, S. Erkki, G. Tion. Mobile technologies in support of distance learning. Asian Journal of Distance Education, v. 1, n. 1, p. 6068, 2003.

[7] L. Martins. M-learning: Aprendizado Móvel na nova Economia Digital. 2006.

Http://pt.shvoong.com/internet-andtechnologies/473562-learning-aprendizadomóvel-nanova/

[8] D. S. Metcalf, J. M. D. Marco. m-Learning: mobile learning and performance in the palm of your hand. Amherst, Ma, Canada: HRD Press, Inc, 2006.

[9] D. Keegan. The Incorporation of Mobile Learning into Mainstream Education and Training. In: Proccedings of m-learning 2005-4th World Conference on m-learning, Cape Town. 2005.

[10] S. Castillo, G. Ayala. ARMOLEO: An Architecture for Mobile Learning Objects. IEEE Multidisciplinary Engineering Education Magazine, IEEE Computer Society, Los Alamitos, CA, USA, v. 2, p. 1-4, 2007.

[11] J. S. Brown, A. Collins, P. Duguid. ARMOLEO: An Architecture for Mobile Learning Objects. IEEE Multidisciplinary Engineering Education Magazine, Washington, USA, v. 18, n. 1, p. 3242, 1989.

[12] D. K. Barry. Web Services and Service-Oriented Architectures: The Savvy Manager's Guide. San Francisco, EUA: Morgan Kaufmann Publishers, 2003.

[13] T. Johnson. Desenvolvendo Aplicações com J2me. São Paulo, Brasil: Novatec, 2007.

[14] C. Horstmann. Padrões e Projetos Orientados a Objetos. São Paulo, SP, Brasil: Bookman, 2006.

[15] HILDI. Cine Mobits no site do LWUIT da Sun. 2009. Http://www.mobits.com.br/2009/5/7/cinemobits-no-site-do-lwuit-da-sun.

[16] C. Houser, P. Thornton, D. Kluge. Mobile Learning: Cell Phones and PDAs for Education. In Computers in Education, International Conference on, IEEE Computer Society, Los Alamitos, CA, USA, p. 1149, 2002.

[17] E. Soloway, C. A. Norris, P. Blumenfeld, B. Fishman, J. Krajcik, R. Marx. Log on education: Handheld devices are ready-at-hand. Commun. ACM, 2001: 15-20
[18] D. Filippo, G.C. Barreto, H.Fuks, C.J.P. Lucena. Collaboration in Learning With Mobile

Devices: Tools for Forum Coordination.

Rio de Janeiro: ABED, 3-6,

Setembro, 2006. Disponível em:

http://groupware.les.inf.pucrio.br/publicacoes/20 06.ICDE.Filippo.CoordinationTools.pdf.

Acesso em: 3 de Jan. 2010.

[19] H. Fuks, D. Felippo, C.J.P. Lucena, C.J.P.de. AulaNetM: Extensão do Serviço de

Conferências do AulaNet destinada a usuários de PDAs. In: Anais do Simpósio Brasileiro de Informática na Educação - SBIE, 16, Juiz de Fora, Novembro, 2005. Disponível em:

http://ritv.les.inf.purio.br/publicacoes/2005.SBIE .Filippo.DescricaoExperimentos.Publicada.pdf. Acesso em: 2 de Jan. 2010.

[20] V. F. Bartholo, M. A. Amaral, M. I. Cagnin. Uma Contribuição para a Adaptabilidade de Ambientes Virtuais de Aprendizagem para Dispositivos Móveis. Revista Brasileira de Informática na Educação, v. 17, n. 2, 2009.

[21] T. Hadzilacos, N. Tryfona. Constructive mLearning Environments. In: ICALT'05: Proceedings of the Fifth IEEE International Conference on Advanced Learning Technologies. Washington, DC, USA: IEEE Computer Society, 2005. p. 271-273.

[22] R. Santos, E. Marçal, C. Vidal, R. Andrade, R. Rios. museuM: Uma Aplicação de m-Learning com Realidade Virtual. In: Anais do 11o Congresso Internacional de Educação a Distância. São Leopoldo: Unisinos, RS, BRASIL: [s.n.], 2005.

[23] F. Buschmann, R. Meunier, H. Rohnert, P. Sommerlad, M. Stal, Pattern-Oriented Software Architecture: A System of Patterns. John Wiley e Sons, West Sussex PO19 IUD, England. 1996.

[24] MobiSiteGalore, mobile website builder. Disponível em: http://www.mobisitegalore.com/ Acesso em: 17 de mar. 2010.

[25] Mobile Author Learning. Disponível em: http://www.abjade.com/ Acesso em: 17 de mar. 2010.

[26] D. Filippo, G.C. Barreto, H.Fuks, C.J.P. Lucena. Collaboration in Learning With Mobile Devices: Tools for Forum Coordination. Rio de Janeiro: ABED, páginas 3-6, Setembro, 2006. 